\title{
Structural Aspects at Phase Transitions in Ferroelectric Copolymers of Vinylidenefluoride and Tetrafluoroethylene
}

\author{
V. V. Kochervinskii ${ }^{1}$, N. V. Kozlova ${ }^{1}$, N. P. Bessonova ${ }^{1}$, M. A. Shcherbina ${ }^{1} \&$ A. S. Pavlov ${ }^{1}$ \\ ${ }^{1}$ Karpov Institute of Physical Chemistry, Vorontsovo Pole 10, Moscow, Russia \\ Correspondence: V. V. Kochervinskii, Karpov Institute of Physical Chemistry, Vorontsovo Pole 10, Moscow \\ 105064, Russia. E-mail: kochval@mail.ru
}

Received: October 28, 2013 Accepted: November 12, 2013 Online Published: March 8, 2014

doi:10.5539/jmsr.v3n2p59 URL: http://dx.doi.org/10.5539/jmsr.v3n2p59

\begin{abstract}
Permittivity and conductivity low-voltage temperature dependences of a ferroelectric copolymer of vinylidenefluoride VDF with tetrafluoroethylene TFE in the ferroelectric to paraelectric phase transition range were investigated. The as received films obtained by crystallization from a solution had the metastable structure shown in particular in coexistence of ferroelectric and paraelectric phases below a Curie point. Temperature dependences of electrical characteristics in the first and second cycles of heating differ. Low glass transition ( -40 ${ }^{\circ} \mathrm{C}$ ) facilitate intensive micro-Brownian chains mobility in the amorphous phase at ambient temperatures and above. As per the $\mathrm{x}$-ray and spectroscopic data, the first heating cycle will result in a recrystallization process for molecular structure improvement. Asymmetry of temperature dependence of a dielectric susceptibility in a vicinity of "order-disorder" phase change is found out. For the first time paid attention to the fact that for crystalline polymer ferroelectrics interpretation of the parameters of the temperature dependences of the dielectric susceptibility in the cycle of heating near the phase transition point should take into account the possibility of the premelt processes. First the method of IR spectroscopy shows that the melting of ordered structures, emerging secondary crystallization is accompanied by a change of the basic parameters (frequency position, the integral intensity and half-width) component of the doublet of valence vibrations of $\mathrm{C}-\mathrm{H}$ groups. Such structural changes lead to the emergence of "anomalies" on the temperature dependences of conductivity.
\end{abstract}

Keywords: Ferroelectric polymers, phase transitions, structure, IR-spectroscopy, conductivity

\section{Introduction}

PVDF-based polymers are known to be favorable object for ferroelectricity investigations in crystalline polymers. The above mention polymers are widely used due to the high piezo-, pyro- (Wang, 1988; Nalva, 1995; Kochervinskii, 1994) and electrostrictive (Kochervinskii, 2003) properties. PVDF is known to have at least up to 4 various crystallographic modifications (Wang, 1988; Nalva, 1995; Kochervinskii, 1996): $\alpha-\beta-\gamma-$ and $\alpha_{p}-$. The first phase has a non-polar cell lattice while the others form crystals with nonzero electrical dipole moment. $\beta$-phase is important for practices, where chain having conformation of a planar zigzag with electrical dipole moment of a monomer unit (2,1 D) being perpendicular to a macromolecule axis (Kochervinskii, 1996). At low temperatures uniaxial drawing of homopolymer is usually causes polymorphic transformation $\alpha \rightarrow \beta$ (Wang, 1988; Nalva, 1995). The isotropic $\beta$-phase films can be prepared by the way of its copolymerization with tetrafluoroethylene TFE or trifluoroethylene TrFE. In this case films can be received by melt or solution crystallization with a polycrystal texture (Wang, 1988; Nalva, 1995; Kochervinskii, 1996): Such materials routinely have zero remnant polarization $\mathrm{P}_{\mathrm{r}}$ and do not show piezo - and pyro-activity. Polarization process was carried out in high electric fields to induce remnant polarization.

The phase transition ferroelectric to paraelectric mechanism research is usually done on copolymers $\mathrm{P}(\mathrm{VDF}-\mathrm{TrFE})$, in which Curie point $\mathrm{T}_{\mathrm{c}}$ and melting point $\mathrm{T}_{\mathrm{m}}$ considerably differ (Wang, 1988; Nalva, 1995; Kochervinskii, 1999) more often. In this paper, the order-disorder transition is investigated on copolymer VDF-TFE of composition 71/29 for which both a chain chemical microstructure (Kochervinskii, 1991), and crystallographic parameters (Kochervinskii, 2010) had being studied earlier. It was shown, that in the copolymer isotropic films been crystallized from a low-boiling solvent at room temperature, $T_{c}$ and $T_{m}$ temperate values differ slightly. It was founded out, that the order-disorder transition having the first kind phase transition type. The electric parameter anomalies in the close proximity to a ferroelectric-paraelectric transition are depends on 
thermal history. The recrystallization processes play important role in transition which may occure in metastable structure films.

\section{Samples and Measurement Techniques}

Investigated random copolymer VDF-TFE contained 29 mol. \% of TFE component with "head to head" HHTT chemical defects 2.5 mol. \% (Kochervinskii, 1991). Copolymer chains contain about 5 mol. \% of diad form TFE comonomer units. To make the film homogenous by thickness the above substrate was aligned horizontal. The samples thickness being $50 \pm 1$ microns.

Wide angle X-ray diffraction patterns (WAXS) were obtained by the powder diffractometer Bruker D8 Advance with quartz monochromater $\left(\mathrm{CuK}_{\alpha}\right.$ radiation, $\left.\lambda=0.154 \mathrm{~nm}\right)$. Volume fractions of various types of crystallites are proportional to the integral intensity of corresponding X-ray scattering. Using spherical coordinates in the reciprocal space, the integral intensity $E$ of scattering intensity can be written as:

$$
E=\iiint I(s, \phi, \varepsilon) s^{2} d s d \varepsilon \sin \phi d \phi
$$

where $I$ is reflection intensity measured at the azimuth angle $\phi, s=\frac{2 \sin \theta}{\lambda}$ is scattering vector, $2 \theta$ is scattering angle, $\lambda$ is X-ray radiation wavelength, and $\varepsilon$ is polar angle. Azimuthal distribution of reflection intensity was measured using a sufficiently large slit width, which ensured the instrumental integration with respect to $d s$. Thus, the measurements of the integral intensity components in the coordinates of $I * \mathrm{~s}^{2}$ versus $\mathrm{s}$ allow the crystal volume fractions to be determined.

Fusion and crystallization processes were investigated by differential scanning calorimetry (DSC) and IR spectroscopy. Perkin-Elmer DSC-7 was used. The heating rate of samples was $2 \%$ min. Calibration was carried out by In. IR spectra were obtained by spectrometer Perkin-Elmer (model 580) with resolution of $2 \mathrm{~cm}^{-1}$. Temperature measurements were taken in a heated cell. This model is used to take into account thermal radiation of the sample. The convolution of the overlapping absorption bands on separate components was carried out by standard program by fitting to experimental points.

To determine the transition Curie point temperature dependences of permittivity and conductivity were used on frequency $1 \mathrm{kHz}$ at heating (cooling) rate of $2 \%$ minute. Al electrodes (thickness $100 \mathrm{~nm}$ ) deposited spattered by vacuum evaporation.

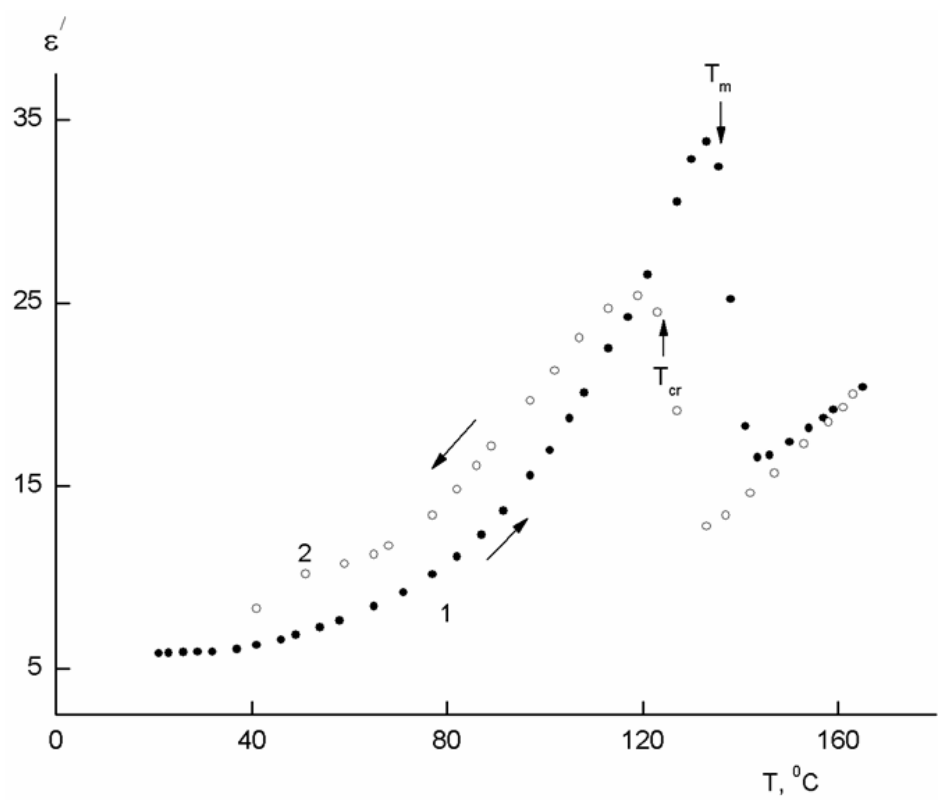

Figure 1. Temperature dependences of the linear dielectric permittivity in film of copolymer VDF-TFE in first cycle of heating (1) - cooling (2); $\mathrm{f}=1 \mathrm{kHz}$ 


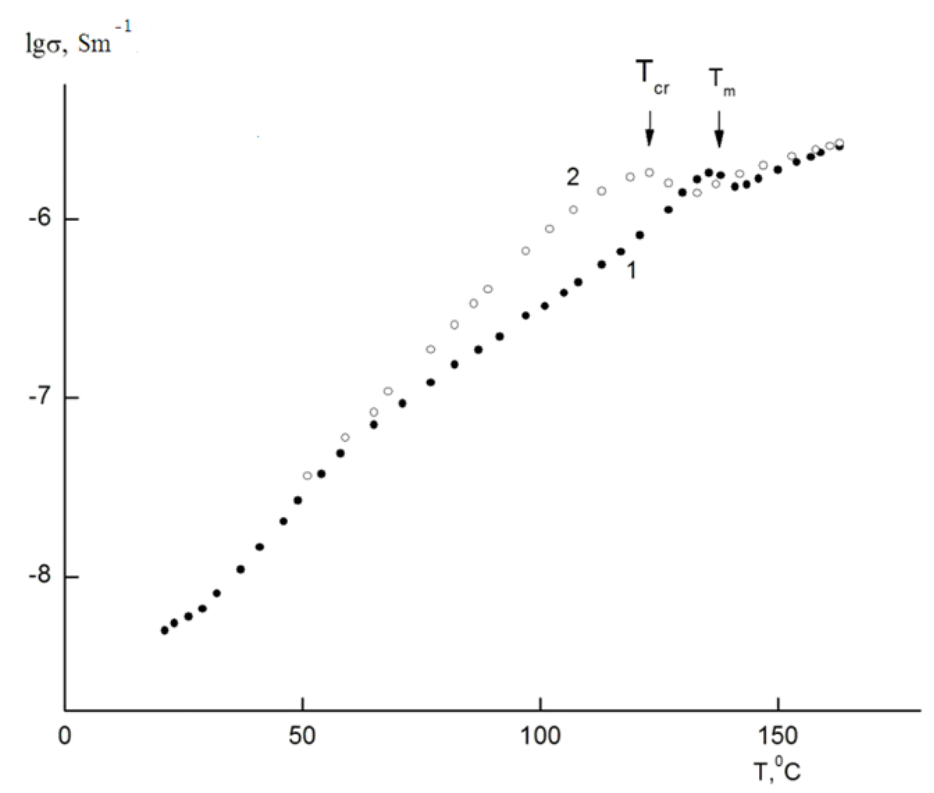

Figure 2. Temperature dependences of the conductivity in film of copolymer VDF-TFE in first cycle of heating (1) - cooling (2); $\mathrm{f}=1 \mathrm{kHz}$

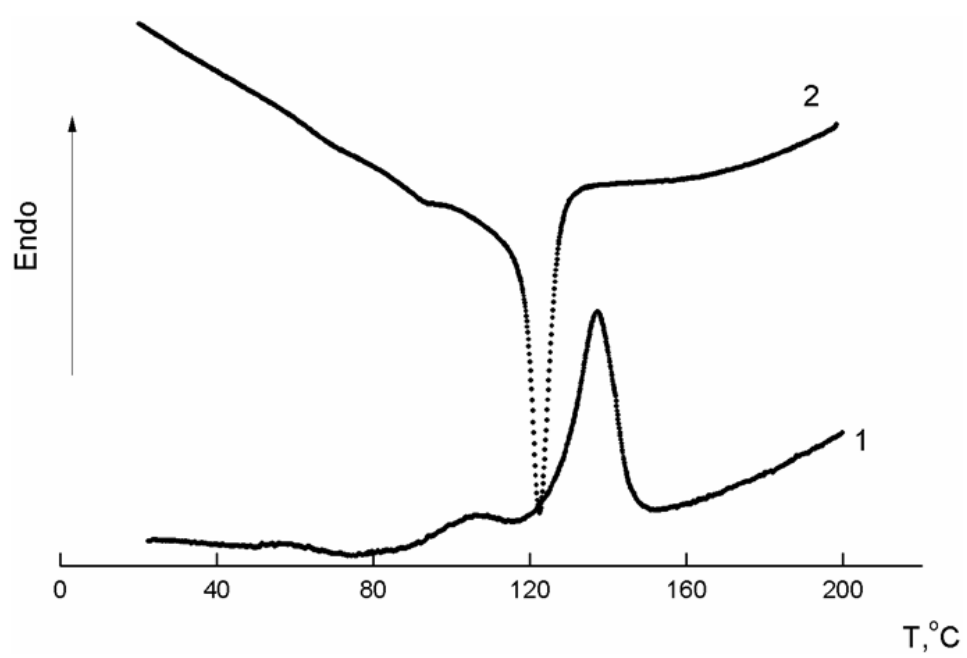

Figure 3. DSC endoderm's in first cycle of heating (1) - cooling (2) in initial film

\section{The Experimental Results}

In Figures 1, 2 temperature dependences of a real part of the linear permittivity $\varepsilon^{\prime}$ and a specific conductivity $\sigma^{\prime}$ in the as received film first heating-cooling cycle are shown. DSK cures obtained at the same heating-cooling mode are plotted in Figure 3. At least two melting-crystallization points may exist. Crystallization temperature $\mathrm{T}_{\mathrm{cr}}$ is lower than melting point $\mathrm{T}_{\mathrm{m}}$ as it is the case with the polymers. Comparison of the Figures 1 and 3 data shows that the dielectric anomalies connected with passing $\varepsilon$ ' through a maximum occur in close proximity to the heat absorption base maximum. The above copolymers are considered to be a ferroelectric material (Wang, 1988; Nalva, 1995; Kochervinskii, 1999, 2010) and this anomalies testifies to a ferroelectric-paraelectric transition. As per Figure 2, $\sigma$ ' anomalies behavior is traced in the proximity of above transition also. 


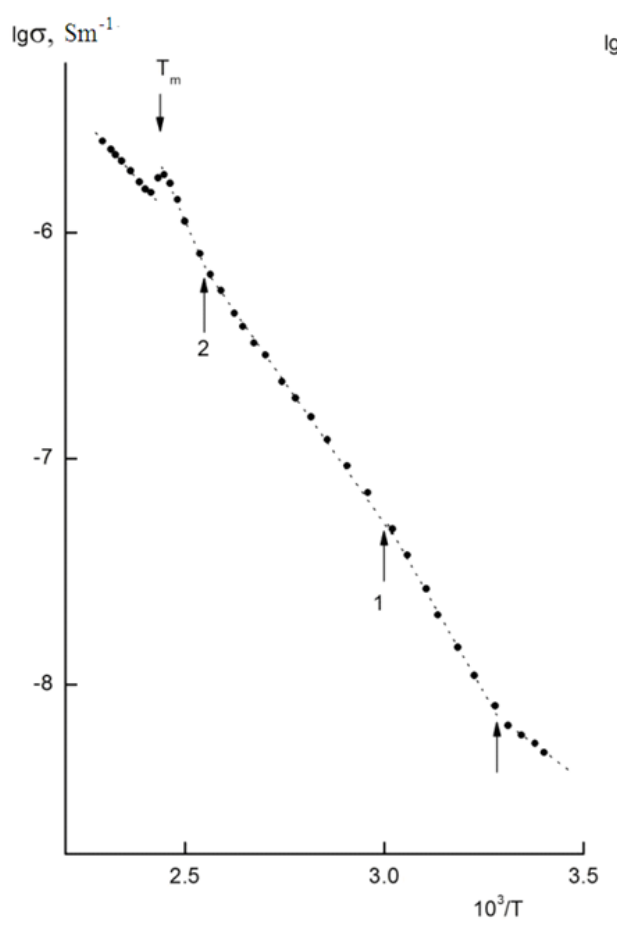

a)

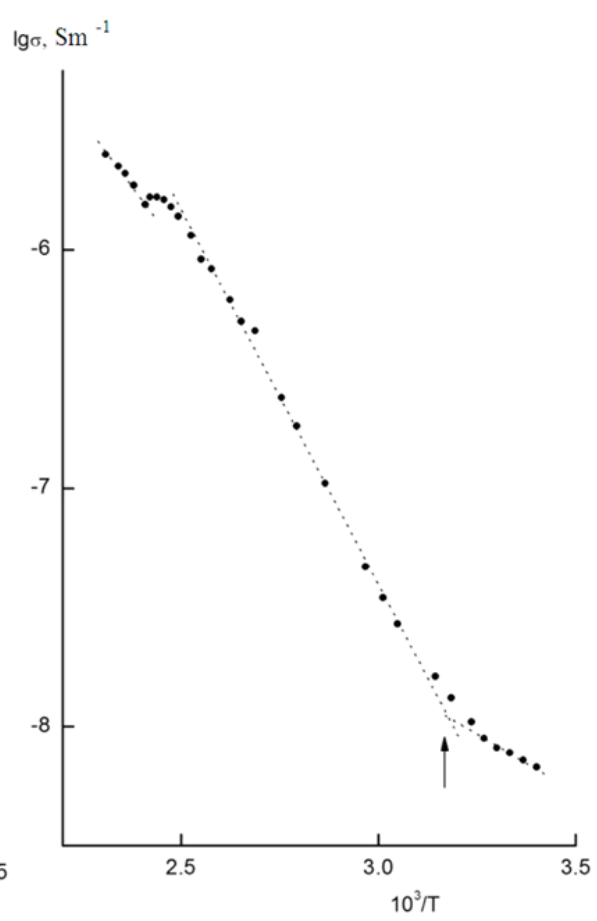

b)

Figure 4. Temperature dependences of the conductivity in coordinates of Arrenius in first (a) and second (b) cycles of heating

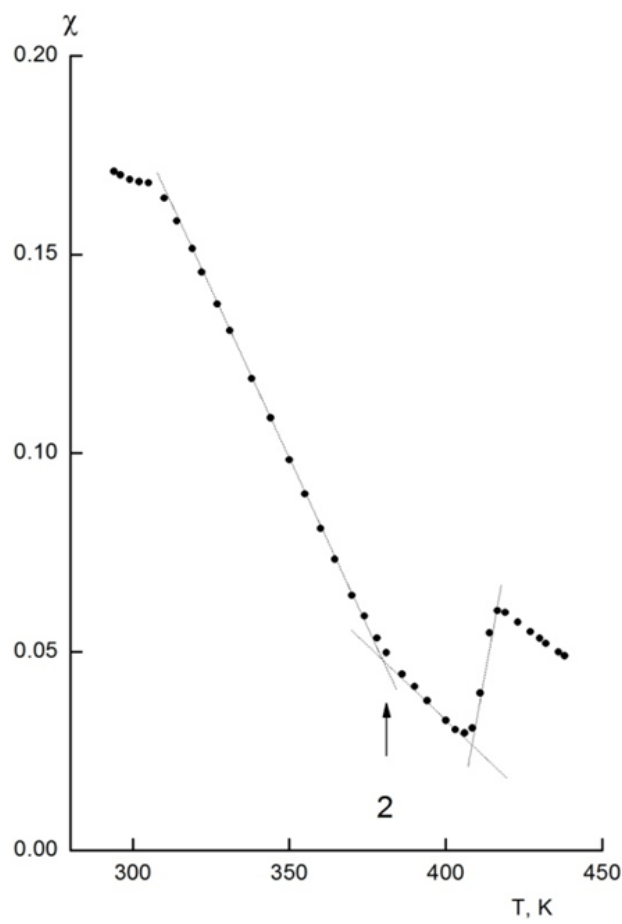

a)

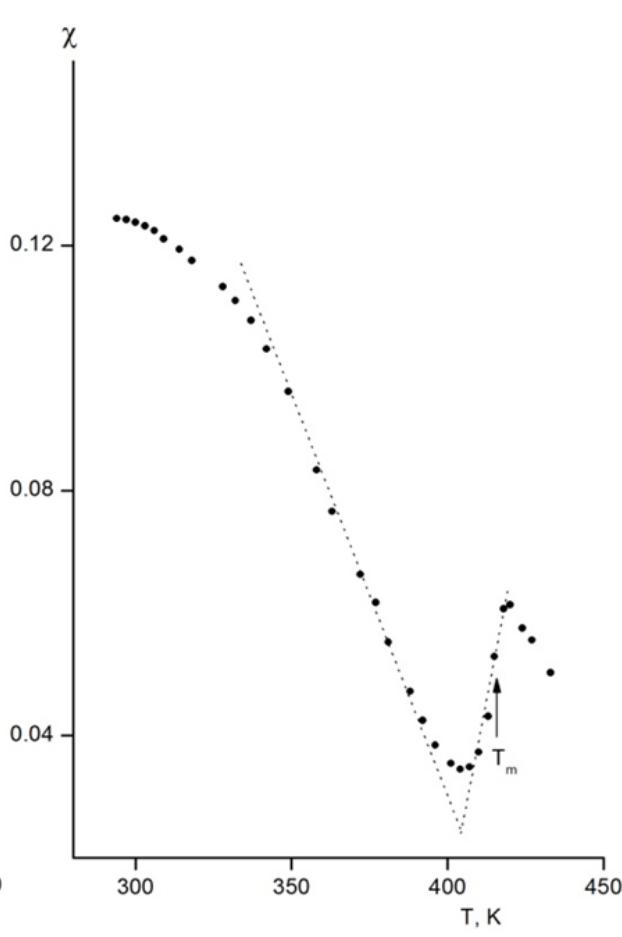

b)

Figure 5. Temperature dependences of the linear dielectric permittivity in first (a) and second (b) cycles of heating

In detail, the curves were analyzed in Arrhenius coordinates (Figure 4). One can see that in the first heating cycle of an as received film (Figure 4a) on the curve there occur a number of distinctive points, showing notable 
changes in the dependences slope. Qualitatively similar dependences have been reported in (Koizumi, 1984). The $\sigma$ ' temperature dependence becomes linear in the second heating cycle (Figure $4 \mathrm{~b}$ ). If $\varepsilon$ ' and $\sigma$ ' temperature changes follow ferroelectric-paraelectric transition, then the Curie-Veiss law is noted to be as:

$$
\chi \approx\left(T_{t}-T\right) / C_{1} \quad \text { at } T<T_{\mathrm{t}} \text { and } \quad \chi \approx\left(T-T_{t}\right) / C_{2} \text { at } T>T_{\mathrm{t}}
$$

where $\chi$ - the linear dielectric susceptibility, $T$ - temperature, $T_{\mathrm{t}}$ - a transition point, and $C_{1}$ and $C_{2}-$ constants.

In Figure 5, $\chi$ temperature dependences for the first and second heating cycle of the as received sample have been presented. Two features were specified. The former being resulted from the fact that $C_{1}$ and $C_{2}$ constants being not identical, i.e. curves being asymmetric. The later can be shown on a curve in the first heating cycle (Figure 5a). In area below a Curie point at temperature $\approx 380 \mathrm{~K}$ (point 2 ) there appear a notable changes of the curve slope, i.e. constant $\mathrm{C}_{1}$ raises. As appears from Figure $5 \mathrm{~b}$, it is not observed for the second heating cycle.

\section{Discussion}

Thus, ferroelectric copolymer films electric characteristics have complex temperature dependences and essentially differ in the first and second heating cycle. In this connection, it is necessary to find out structural changes in the course of heating process, and irreversible changes in a structure film after the first heating cycle. It is known, that crystalline polymers are objects, predisposed to form metastable states. It is caused by the chain nature of molecules and polymers morphology variety. In the present work the chosen way of films preparation facilitates the formation of above metastability. The crystallization was carried out at room temperature from acetone which unlike dimethylformamide DMFA or dimethylsulfoxide DMSO (Shapiro, 1982) does not possess high affinity to the polymer. As result, this leads the crystallization proceeds in the conditions of high overcooling. For PVDF and its copolymers are studied crystallization temperature affects characteristics and transitions in them. For example, crystallization temperature decrease in VDF-TrFE results in secondary transitions temperatures increase (Oka, 1986; Tanaka, 1988; Rinaldo, 1988). Qualitatively similar situation is observed in our case as well. In the second heating cycle the noted transition is shifted to lower temperatures (Figure 6). The recrystallization leads the system to more equilibrium polymer state after first heating of the metastable structure sample. The left insert to Figure 6 confirms such hypothesis. The basic melting peak at first approximation is characterized by three components. Conditionally, they can referred to three types of the crystals differing in the sizes and (or) degree of perfection. The components intensities ratios changes in the second heating cycle. Recrystallization process (in the first heating cycle) is followed by formation of more perfect (or larger size) crystals. X-ray diffraction method was used to prove it. Our data as well as that of other authors (Wang, 1988; Nalva, 1995; Kochervinskii, 1996) show that copolymer crystallized into polymorphic $\beta$-phase at given conditions (Kochervinskii, 2010). For this phase peak positions from planes 110 and 200 for copolymers VDF coincide (Wang, 1988; Nalva, 1995; Kochervinskii, 1996). In the case of an as received film (Figure 7a) the best coincidence of the experimental curve with rated one appears in the presence of two components. As appears from Figure $7 \mathrm{~b}$, annealing in the continuous heating mode to $200{ }^{\circ} \mathrm{C}$ is accompanied by appreciable change of diffraction curves, especially for intramolecular order (larger s). As it is earlier noted on copolymers VDF with hexafluoropropylene (Kochervinskii, 2011), the additional peaks are observed in a film after recrystallization. The changes occur in some structural parameters after the recrystallization in as shown in the table. After recrystallization the size of crystals along planes 200 and 110 rises. It can occurs at partial transition of a paraelectric phase (which we refer to a peak-halo at $2 \theta \approx 18^{\circ}$ (Kochervinskii, 2010)) to a ferroelectric phase, (the peaks 200 and 110). Really, the size of crystals of a paraelectric phase $\left(1_{p}\right)$ in a film after recrystallization decreases (table). It is confirmed by decrease of paraelectric phase fraction $\left(\alpha_{p}\right)$ in the given film. The $\alpha_{\mathrm{p}}$ are calculated as follows

$$
\alpha_{p}=\frac{I_{p}}{I_{p}+I_{200}+I_{110}}
$$

where $I_{p}, I_{200}, I_{110}$ - integral intensity peaks of paraelectric and ferroelectric phases respectively.

Table 1. Structural parameters in as received film and after recrystallization one

\begin{tabular}{llllllll}
\hline Parameter & $1_{200}, \mathrm{~nm}$ & $1_{110}, \mathrm{~nm}$ & $1_{\mathrm{p}}, \mathrm{nm}$ & $\alpha_{\mathrm{c}}$ & $\alpha_{\mathrm{p}}$ & $\mathrm{D}_{822}+\mathrm{D}_{834} / \mathrm{D}_{840}$ & $\mathrm{D}_{905} / \mathrm{D}_{840}$ \\
\hline As received & 7.4 & 11.8 & 2.8 & 0.29 & 0.59 & 1.36 & 1.31 \\
After recrystallization & 7.6 & 17.1 & 2.1 & 0.31 & 0.47 & 0.98 & 0.76 \\
\hline
\end{tabular}


The table shows that films after recrystallization have a tendency to the crystallinity degree rize, obtained by $\mathrm{X}$-ray method $\left(\alpha_{\mathrm{c}}\right)$. The data of IR-spectroscopy also proves this conclusion. compared The ratio of the integrated intensities of the bands "vagueness" $\left(905 \mathrm{~cm}^{-1}\right)$ and "crystallinity" $\left(840 \mathrm{~cm}^{-1}\right)$ was compared. From the last column of the table shows that in recrystallizated film the ratio decreases.

a)
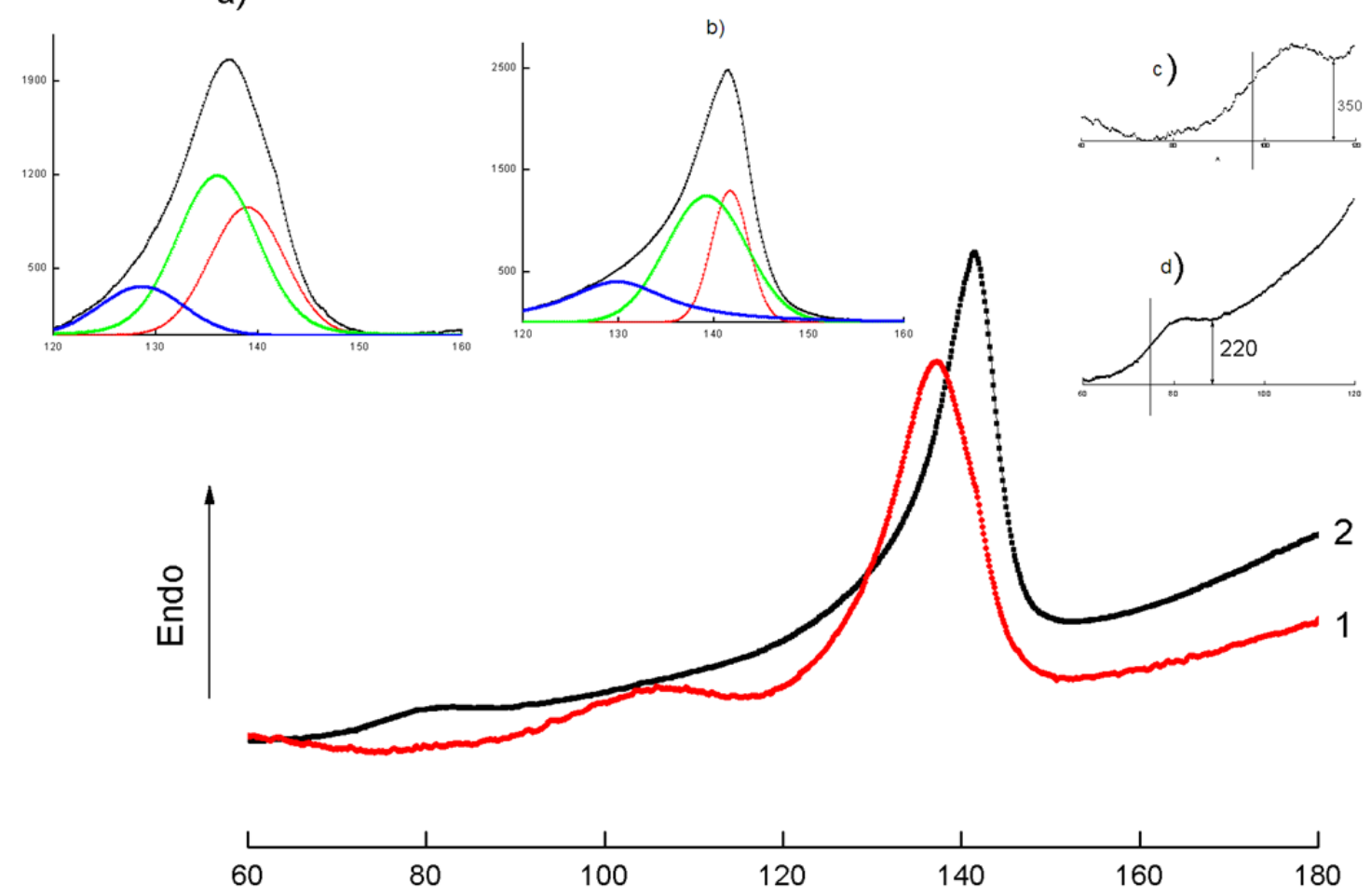

T, C

Figure 6. DSC endoderm's in first $(1, \mathrm{a}, \mathrm{c})$ and second $(2, \mathrm{~b}, \mathrm{~d})$ heating cycles. Insert: scheme deconvolution (a, b) of curves for main melting peak and curves of heat flow for second transition (c, d)

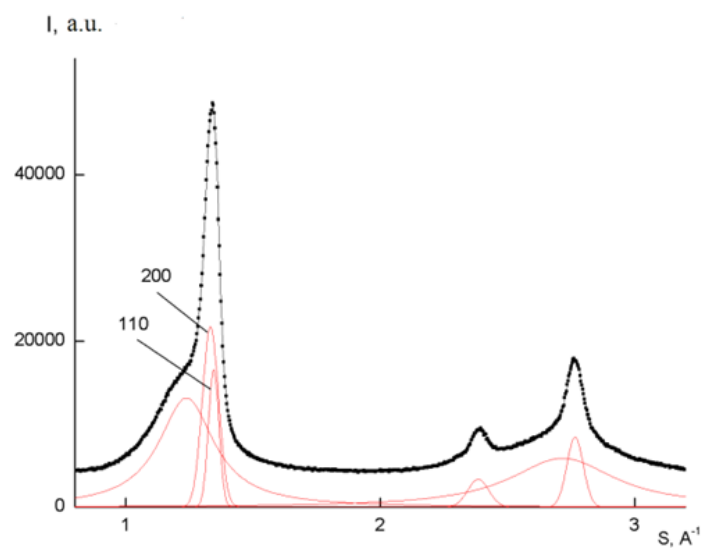

a)

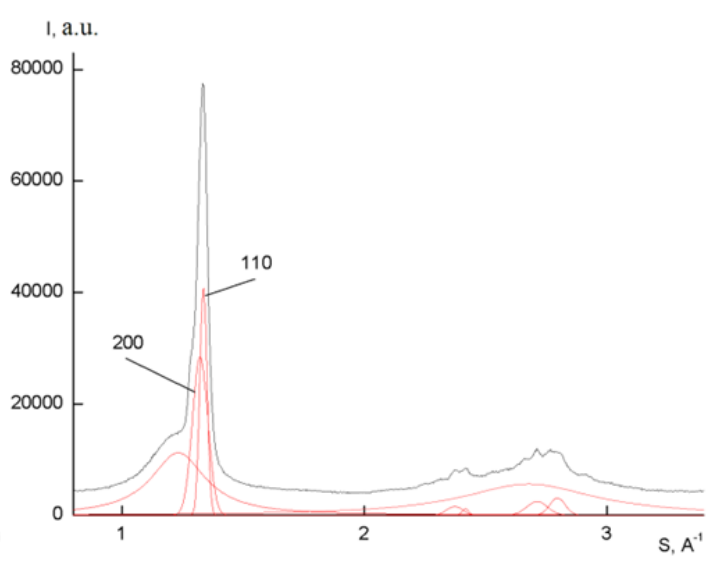

b)

Figure 7. X-ray dates of the films; (a) - as received (b) - after recrystallization

IR spectroscopy data (partially they are presented on Figure 8) confirm x-ray method conclusions. Chains in a

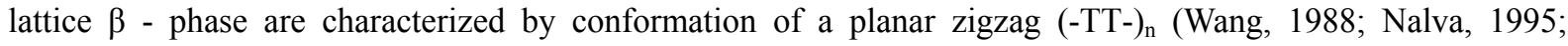


Kochervinskii, 1996). The absorption peaks $447,509,840,1275 \mathrm{~cm}^{-1}$ are characteristic of this conformation. The analysis shows presence of the isomers in the form of TG $\left(490 \mathrm{~cm}^{-1}\right)$, TGTG $-\left(410,530,765\right.$, and $\left.795 \mathrm{~cm}^{-1}\right)$ and $\mathrm{T}_{3} \mathrm{GT}_{3} \mathrm{G}^{-}-\left(420,430,822,834 \mathrm{~cm}^{-1}\right)$ (Wang, 1988; Nalva, 1995; Kochervinskii, 1996; Kobayashi, 1975; Tashiko, 1981; Lovinger, 1988). Chains in these conformations can exist in an amorphous phase, on boundary of an amorphous - crystal phase and in crystals of a paraelectric phase. Both paraelectric and ferroelectric phase can be coexistence at temperature ranges below a Curie point in the given class of polymers (Wang, 1988; Nalva, 1995; Kochervinskii, 1999; Horiuchi, 1986). Antiferroelectric phase (being registered by dielectric hysteresis curves (Kochervinskii, 1991; Takahashi, 1999)) can contain the "defect" conformations chains also. The spectrum range, sensitive to the presence of long $(\mathrm{n} \geq 3)$ chain sequences in a planar zigzag conformation $\left(840 \mathrm{~cm}^{-1}\right)$ is shown on Figure 8 . The bands of $822,834 \mathrm{~cm}^{-1}$, that is referred to conformation $\mathrm{T}_{3} \mathrm{GT}_{3} \mathrm{G}$, (Kobayashi, 1975; Tashiko, 1981; Lovinger, 1988) is also observed. The ratio of these isomers has been calculated by the integrated intensities equation $D_{822}+D_{834} / \mathrm{D}_{840}$. As it is clear from the table, the recrystallization process is followed by its decrease. Thus, the recrystallization process is accompanied by conformation transitions $T_{3} G_{3} G-\rightarrow(-T T-)_{n}$. It should be remembered, that the chains in a paraelectric phase have conformation $\mathrm{T}_{3} \mathrm{GT}_{3} \mathrm{G}^{-}$also (Wang, 1988; Nalva, 1995; Kochervinskii, 1999). Thus, the above conformation changes during recrystallization result in the polar crystals size increase.

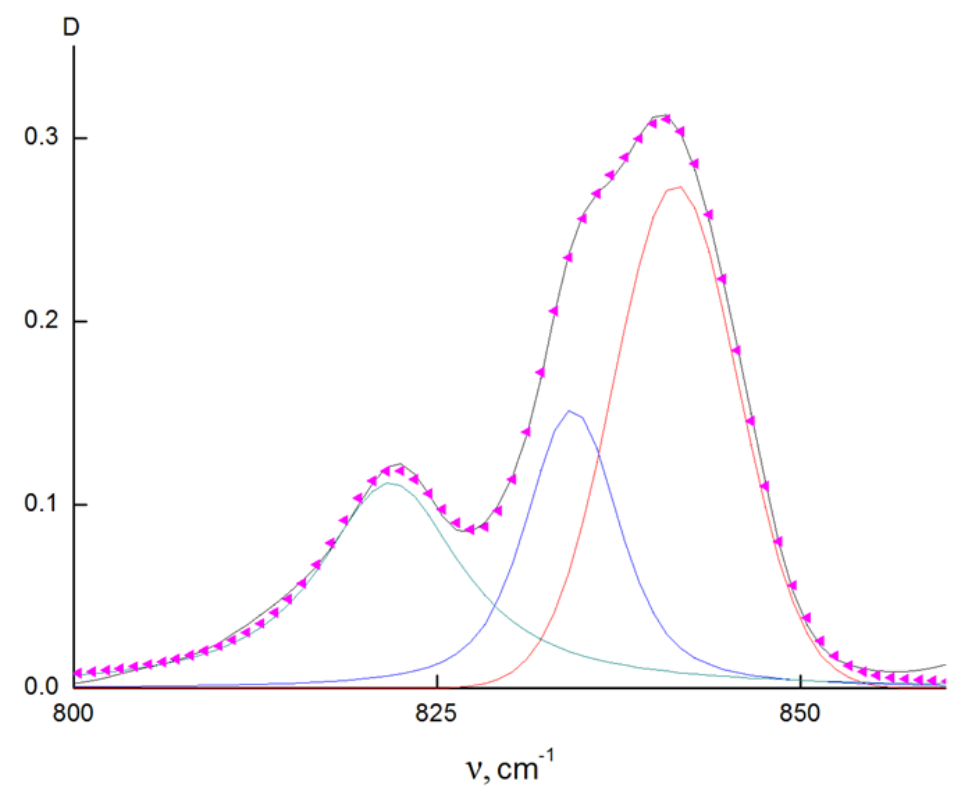

Figure 8. IR spectra of the initial film for vibrations, which to be responsive to specific conformation of molecular groups

It is necessary to consider the reasons causing the "abnormal" displacement of secondary transition to lower temperatures after recrystallization. Relaxation processes in this transition may follow two mechanisms. The former occur in a rigid amorphous phase (RAP) molecular mobility. Provided that the secondary transition has to do with the RAP relaxation, this phase ratio will decrease after the first heating cycle. With the internal stresses in RAP chains decrease the second heating cycle transition area changes to lower temperatures. The latter is secondary crystallization imperfect crystals melting, which is referred to (Neidhoffer, 2004). In this case after the first heating (annealing) cycle the secondary crystallization melting peak decrease due to the kinetic reasons. Actually, an as received film after fabrication has been stored at room temperature for several years. At low glass transition $\left(-40^{\circ} \mathrm{C}\right)$ such conditions (equivalent to long annealing) can result in a larger ratio and higher perfection of secondary crystals formed.

As noted above, the ferroelectric-paraelectric transition is characterized by the difference in Curie constants $\mathrm{C}_{1}$ and $\mathrm{C}_{2}$. A qualitatively similar phenomenon was noted, for example, in the copolymer VDF/TrFE (Miyata, 1988). In our case, the $C_{1}$ value change (Figure 5a) near the Curie point can be noted in the first heating cycle. We consider this fact to be connected with structural changes following the recrystallization near this point in as received metastable films. But even in the second heating cycle $\chi(T)$ curve asymmetry still remains, as it is clear from comparison of Figures $5 \mathrm{a}$ to $5 \mathrm{~b}$. A theoretical analysis of the linear permittivity constant behavior in these 
polymers near the phase transition is given in (Ikeda, 1984). The three stable states: $1,-1$, and 0 , system can be taken as the model. It is known to be typical for antiferroelectrics. Our polymers antiferroelectrical properties are noted in (Kochervinskii, 1991; Takahashi, 1999). As per this theory, the $\varepsilon$ ' value determined by the M fluctuations magnitude of a volume $\mathrm{V}$ dielectric material dipole moment can be expressed as follows:

$$
\varepsilon^{\prime}-1=\frac{\left\langle\left(M-\langle M\rangle^{2}\right\rangle\right.}{\varepsilon_{0} V k T}
$$

where $\varepsilon_{0}$ - the vacuum permittivity, $k$ - the Boltzmann constant and $T$ - the temperature, the angle brackets denote statistical averaging operation.

It was assumed, that $M$ is the vector sum of dipole moments of monomer units $\mu_{\mathrm{i}}$, where the relation $\mu_{\mathrm{i}}=\alpha_{\mathrm{i}} \mu\left(\alpha_{\mathrm{i}}\right.$ $=1,-1,0)$. Given that $\varepsilon \gg 1$ the final expression for $\varepsilon$ ' has the form (Ikeda, 1984):

$$
\varepsilon^{\prime}=G \eta \frac{N \mu^{2}}{\varepsilon_{0} k T}
$$

where $G$ and $\eta$ - some function defining the probability of states $(1,-1,0), N$ - total number of dipoles per unit volume $\left(N=N_{1}+N_{-1}+N_{0}\right)$. In accordance with (5) the $\varepsilon$ ' temperature dependence near Curie point will be determined predominantly by the functions $G$ and $\eta$. Above theory is applicable to crystalline systems. The investigated polymers belong to a partially crystalline class, where the crystallinephase can equal 50 percent or less (Wang, 1988; Nalva, 1995; Kochervinskii, 2010). Micro-Brownian mobility presence in the amorphous phase will result in the $\varepsilon$ ' increase. Crystal's distribution by size affects the permittivity temperature dependence. In this connection, DSC curve low-temperature asymmetrie scan be attributed to small and imperfect crystallites melting process (premelting) (Figure 6). This process will naturally be accompanied by a decrease in crystallinity and $N$ (Equation (5)) is a temperature decreasing function. Considering this fact, the $\varepsilon$ ' temperature dependence in the phase transition proximity will be determined not only by the functions $G(T)$ and $\eta(T)$, but by a character $N(T)$ curve as well. This qualitatively explains the asymmetry of the $\chi(T)$ curves and therefore constants $\mathrm{C}_{1}$ and $\mathrm{C}_{2}$ inequality in (2).

As noted above (Figure 4), in the first heating cycle (where the original film with a metastable structure is brought to more the equilibrium state), there is a number of specific points where slope angle changes. A hopping transport mechanism $\sigma$ temperature dependence is assume to be described by

$$
\sigma=\sigma_{0} \exp (-\Delta E / k T)
$$

where $\sigma_{0}-$ preexponential factor, $\Delta E$ - energy activation.

It means that there is an activation energy decrease point 1 (Figure 4a). Secondary transition is observed to occur exactly in this temperature range (Figures 3,6). In our opinion, above secondary transition will result in the lowering of the "free" charges mobility activation energy, which is proved by the experiment. The activation energy is shown to rise in point 2 with temperature rise (Figure 4a). The main melting peak has been registered in this temperature range. This process will be accompanied by a simultaneous process of recrystallization. The more perfect crystals at the same time are formed, the deeper traps for carriers will be. It will be followed by conductivity activation energy increase (Figure 4a). By comparison of Figures $4 \mathrm{a}$ and $5 \mathrm{a}$ it is clear, that the temperature at points 2 for both curves coincide. This means that the temperatures, where the observed "anomalous" changes in the activation energy of conductivity and the constant $\mathrm{C}_{1}$, are the same. By comparison of Figure $4 b$ to $5 b$ it is clear, that there is a linear relation of both conductivity and dielectric susceptibility in a recrystallized film at temperatures above $60^{\circ}$ which proves the above.

The real polymer materials expose weak electrolyte properties. One of which being that impurities, contained in the polymer, having either salt, or acid, or alkaline origin. The analyzed objects refer to crystalline polymers, therefore impurities must be localized in the amorphous phase, which has a high "free volume" and a high dielectric constant (Kochervinskii, 1996). As is the case with the electrolytes, the impurities will dissociate to form ions involved in the charge transfer process of an electric field. Obviously, the dissociation level and therefore the $\mathrm{n}$ carrier concentration will depend on $\varepsilon$ ' environment. If the latter increases with increasing temperature (Figure 1), in accordance with the foregoing, the carrier's concentration in the system will increase as well. The comparison of Figures 1 and 2 proves the fact that the conductivity in a narrow temperature range in the areas above the Curie point (where the dielectric constant begins to decrees), is "abnormally" reduced as well.

For more detailed structural changes analysis in the as resived films recrystallization process the data on the IR spectra temperature dependence was used. A number of high-frequency bands, which are fairly easy to analyze, 
was reported to be in the investigated polymers. The absorption bands referred to symmetric and antisymmetric stretching vibrations of methylene groups were selected (Wang, 1988; Nalva, 1995; Kochervinskii, 1996). The three different temperatures spectra are shown in Figure 9. Additional components for symmetrical $\left(2969 \mathrm{~cm}^{-1}\right)$, and antisymmetrical $\left(3008 \mathrm{~cm}^{-1}\right)$ vibrations can be observed. There is only one work, which refers to the appearance of a fine structure in this spectrum range after PVDF films annealing (Bachmann, 1979). However, this is a fact the authors did not comment upon. Nevertheless, as per reference (Bachmann, 1979), the more ordered the PVDF samples structure is, the finer structure in this doublet will be. Figure 9 shows that intermolecular interactions affect the fine spectra structure as well. The temperature increase (leading to a weakening of such interactions) results in the widening of the above doublet bands. Our analysis of this spectral range for the VDF homopolymer and 94-6 VDF-TFE copolymer shows that the discussed effect manifests itself only in a weak asymmetry of the marked bands. Similar results were observed for the PVDF in (Tashiro, 1983), as well. The wide and small angles X-ray diffraction data shows (Kochervinskii, 2006a, 2006b, 2010), that crystallized films of VDF copolymers with a TFE high content are characterized by large amorphous gaps between the edge surfaces of lamellar crystals. It is the above phenomena connected with the weakening of the intermolecular dipolar interactions that will be responsible for the observed spectral features.

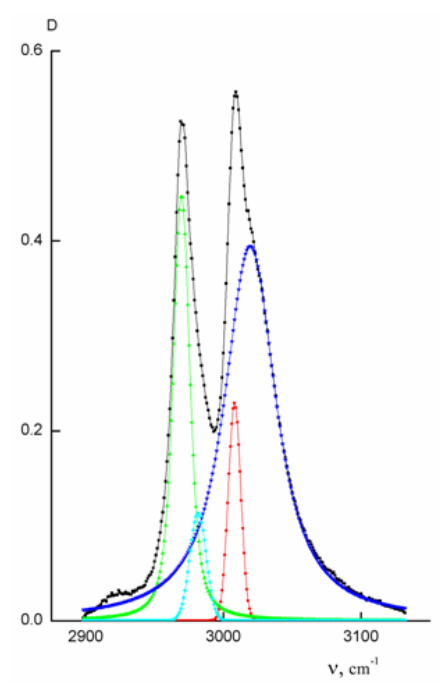

a)

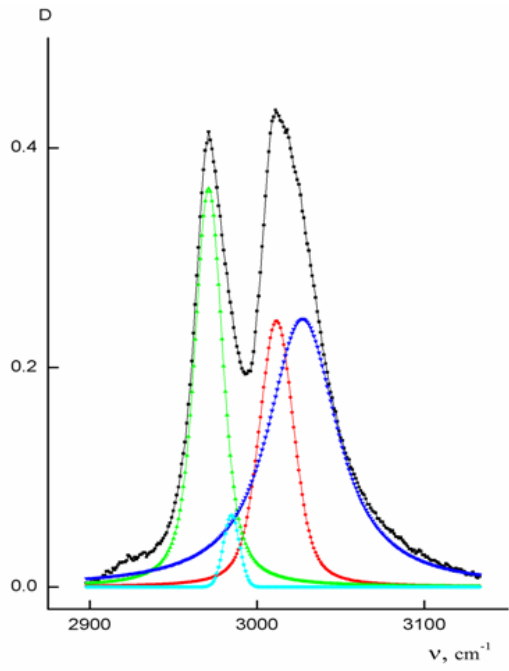

b)

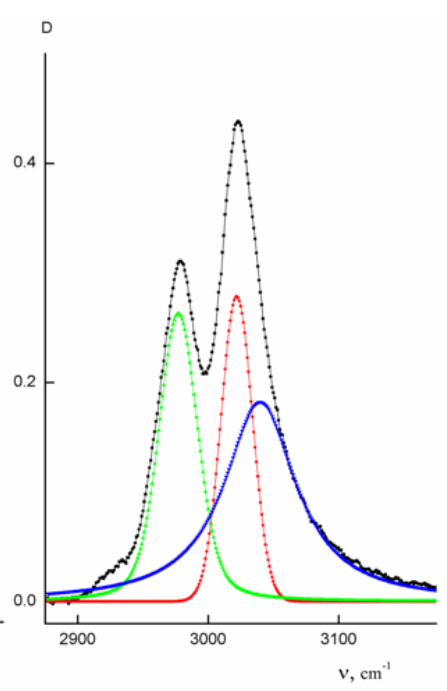

c)

Figure 9. IR spectra's in the region of the stretching vibrations of methylene groups at temperatures: 30 (a), 80 (b) and $130^{\circ} \mathrm{C}(\mathrm{c})$

The analysis of the absorption bands shapes shows that the experimental spectra (some of which are presented in Figure 9) is best described by four components. Each of these components was characterized by the $v_{\mathrm{m}}$ frequency position, $\delta v$ FWHM and the $I_{\mathrm{s}}$ integrated intensity. The temperature dependence for all four components is shown in Figures 10-12. All three characteristic parameters for abovementioned the absorption bands considerably changes in a narrow range of temperatures $\left(70-80{ }^{\circ} \mathrm{C}\right)$. Let's investigate frequency position temperature dependences. It is known that the $\Delta v_{\mathrm{T}}$ frequency shift during temperature increase from $T_{1}$ to $T_{2}$, in other polymers, (Bretzlaff, 1981, 1983; Vettegren, 1992, 2009; Bronnikov, 1996) can be written as follows:

$$
\Delta v_{\mathrm{T}}=v\left(\mathrm{~T}_{2}\right)-v\left(\mathrm{~T}_{1}\right)=\beta_{\mathrm{x}}\left(\mathrm{T}_{2}-\mathrm{T}_{1}\right)
$$

where coefficient $\beta_{\mathrm{x}}$ for backbone vibrations in the polymer usually has a negative value, and its magnitude varies in the occurrence of various relaxation transitions (Vettegren, 1992, 2009; Bronnikov, 1996).

As follows from Figure 10a, in our case, this coefficient has the positive value. One reason for this "discrepancy" being that, we have analyzed the vibrations, other than backbone ones. The chemical structure of the polymer should also manifest itself here, because "blue" heating shift was noted in PVDF for the vibrational bands ( $v_{\mathrm{s}}$ $\mathrm{CF}_{2}+\mathrm{t} \mathrm{CH}_{2}$ ) (Peng, 2004). It is interesting to evaluate the possible role of internal stresses. In multiple nucleations during crystallization from solution, there occur internal stresses in chains along the boundaries of neighboring crystals. This can influence the absorption bands frequency position. Indeed, the application of $\sigma_{\mathrm{m}}$ mechanical stress to the polymer films under isothermal conditions result in a $\Delta v_{\sigma}$ shift of the characteristic 
frequencies of the vibration spectrum as per the following equation (Bretzlaff, 1981, 1983; Vettegren, 1992, 2009; Bronnikov, 1996)

$$
\Delta v_{\sigma}=v\left(\sigma_{\mathrm{m}}\right)-v(0)=\alpha_{\mathrm{x}} \sigma_{\mathrm{m}}
$$

with the $\alpha_{\mathrm{x}}$ coefficient being of negative value. If during heating these stresses are reduced, the bands should shift to higher frequencies. The analysis of the data (Bretzlaff, 1981, 1983; Vettegren, 1992, 2009; Bronnikov, 1996) shows that the "red" shift of the absorption bands under external mechanical stresses in highly oriented polypropylene is less than $3 \mathrm{~cm}^{-1}$ even at scission strain (Bretzlaff, 1981, 1983). In our case the observed bands shift is several times higher (Figure 10). In this regard, the absorption bands "blue" shift increase can occur with temperature rise in this class of polymers due to other reasons as well.

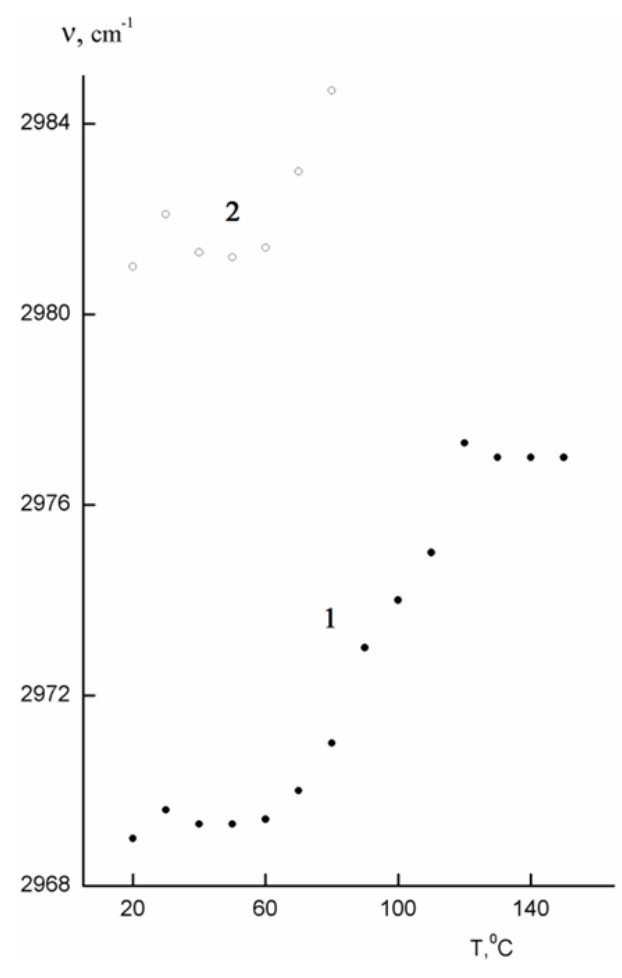

a)

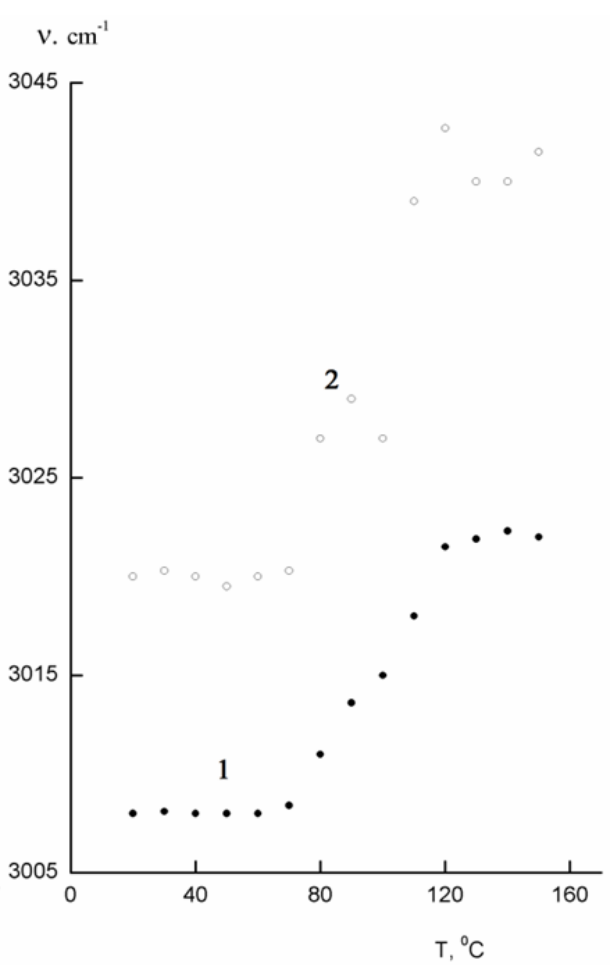

b)

Figure 10. Temperature dependences of peak frequencies for low-frequency (1) and high-frequency (2) components of the symmetric (a) and asymmetric (b) stretching vibration of the methylene groups

The above polymers show structural and dynamic heterogeneity due to the existing of crystalline and amorphous phases. The latter phase may (ratio of that can be up to 0.5 and above (Kochervinskii, 2010)) characterize by the low glass transition temperature $\left(-40^{\circ} \mathrm{C}\right)$. In consequence of this, disordered phase chains at above ambient temperature are involved in the cooperative Brownian motion. Dielectric relaxation data shows that the relaxation time of such mobility in the temperature range are smaller than $10^{-7} \mathrm{c}$ (Kochervinskii, 1996). The $\mathrm{X}$-ray diffraction (Figure 7) pointed out the presence of the halo, which means that the structure of this phase is characterized by the absence of a long-range order. Thus, the structural and dynamic parameters of the amorphous phase make us believe it to be a liquid-like. On the other hand, due to the presence of C-F bonds in the chains with the high dipole moment monomer unit $(2,1 \mathrm{D})$, the electrostatic interactions being a major factor affecting the width and frequency position of the absorption bands (Volkenstein, 1937). For number high-polar liquids parameters of the absorption bands determine by the fluctuations, or Brownian dynamics (Bahshiev, 1972). The process of rotational motion is always accompanied by a broadening of the bands (Rakov, 1962; Valiev, 1961, 1963) and changing of their shape (Bulanin, 1964, 1965). For high-polar liquids (Sechkarev, 1965), $\mathrm{U}(\mathrm{r})$ potential energy function of polyatomic molecules in condensed state is adjusted taking into account the orientation interaction of neighboring molecules $\mathrm{F}(\mathrm{r})$ in the following equation

$$
\mathrm{F}(\mathrm{r})=-\mu \mathrm{E}_{\mathrm{i}} \cos \varphi_{\mathrm{i}}
$$


where $\varphi_{\mathrm{i}}$ - the angle between the dipole moment $\mu$ and the local field of the light wave $E_{\mathrm{i}}$ near the $\mathrm{i}$-th molecule. Than $\Delta \omega$ difference between the angular frequency $\omega$ in condenses state and isolated molecule frequency $\omega_{0}$ is given as follows (Sechkarev, 1965):

$$
\Delta \omega=\omega-\omega_{0}=\frac{\left(\frac{\partial \varphi}{\partial q}\right)^{2}-\frac{1}{\mu} \cdot \frac{\partial^{2} \mu}{\partial q^{2}}}{M \omega_{0}} \cdot \frac{s \mu^{2}}{R^{3}}
$$

where $\mathrm{M}$ - reduced mass, $\mathrm{R}$ - the average distance between neighboring molecules, $\mathrm{s}=\mathrm{s}(\mathrm{T})$ - a parameter of mutual orientation, $\mathrm{q}$ - normal coordinate.

The former term in the numerator of Equation (10) characterizes the change in the dipole orientation and the latter is the change in dipole moment magnitude. As per Equation (10), there may exist both "red" $(\Delta \omega<0)$ and "blue" $(\Delta \omega>0)$ shift of the absorption band. It is determined by the sign and magnitude of the second derivative of the dipole moment by the q normal coordinate. In this case, "blue" shift (Figure 10a) can corresponds to a negative value of the second term in the numerator of Equation (10).

As per ref. (Sechkarev, 1965), the half-width (FWHM) of the absorption band $\delta \omega_{0.5}$ will be determined as

$$
\delta \omega_{0.5}=\Delta v \approx \frac{2 T}{\sqrt{\pi}} \sqrt{\frac{2 k\left(\omega-\omega_{0}\right)}{\bar{V}}\left|\frac{d \omega}{d T}\right|}
$$

where $T$-temperature, $\bar{V}$ - average energy of the orientation interaction.

Equation (11) shows, that there is a term in the square root expression that takes into account an additional contribution to thermal broadening of the bands, due to the temperature shift of the band. An additional broadening is observed in the temperature region where there is a bands "blue" shift, which is proved by comparison of Figures 10 and 11.

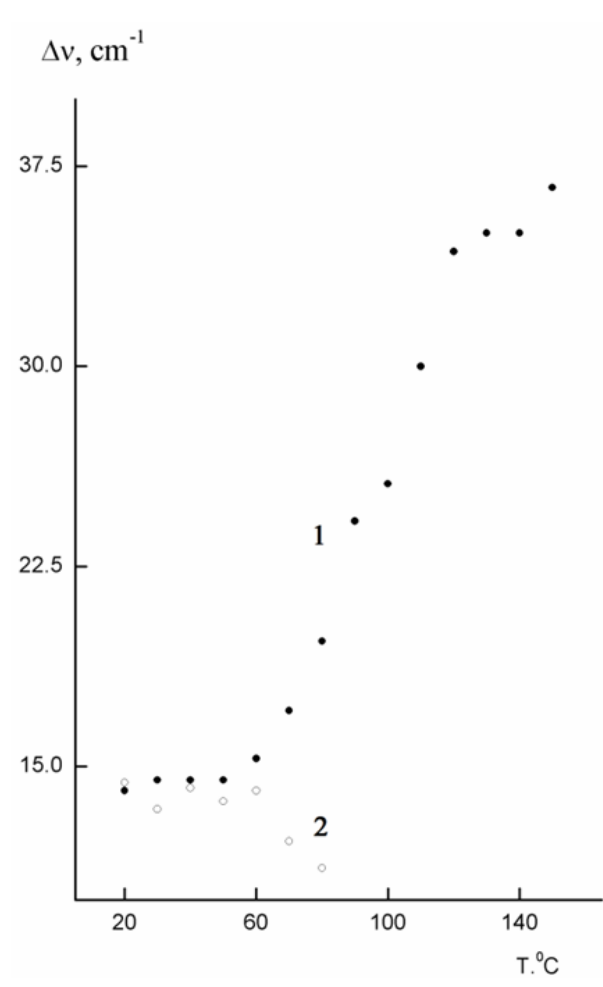

a)

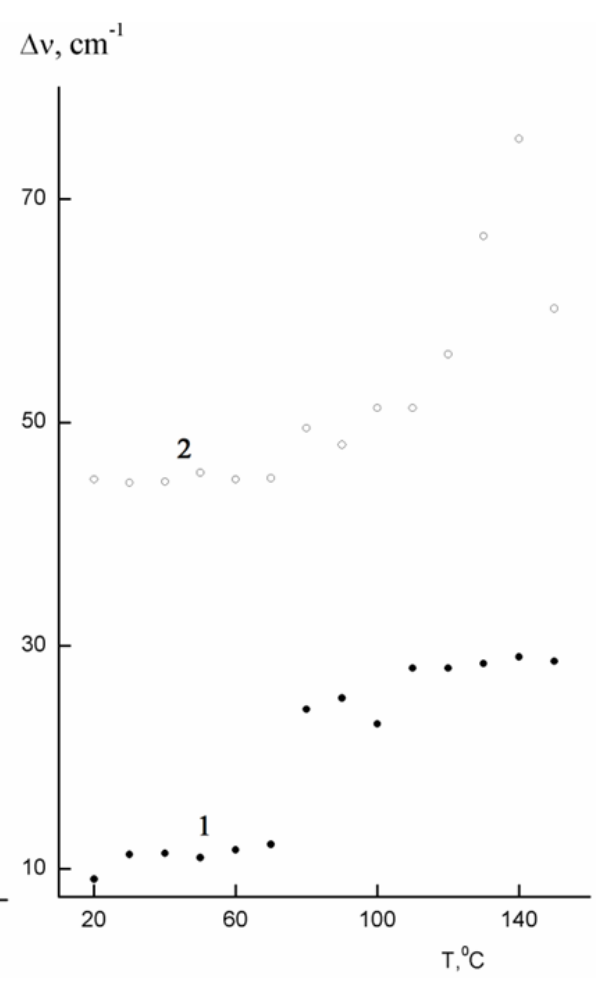

b)

Figure 11. Temperature dependences FWHM of the symmetric (a) and asymmetric (b) stretching vibrations bands; low-frequency (1) and high-frequency (2) components

Dielectric increment $\Delta \varepsilon$ (proportional to the effective dipole moment) is noted to have unusual temperature dependence in above polymers (Kochervinskii, 1996; Nakagawa, 1973). Moreover, it was shown that the 
dielectric increment temperature dependence has a broad maximum (Nakagawa, 1973). It is approximately in the same temperature region, where in our case there is a change in the spectral characteristics of the absorption bands.

According to (Bahshiev, 1972; Rakov, 1962) for high-polar liquids fluctuations microdensity and rotational mobility relaxation should result monotonous change the frequency positions with temperature. As per Figures 10-12, this absorption bands spectral characteristic change occurs in a narrow temperature range for our polymers. It makes us believe that there is a phase transition in this area. This hypothesis is proved by the non-monotonous character of the heat absorption curve (Figure 6) and presence $\Delta \varepsilon(\mathrm{T})$ maximum segmental mobility of the PVDF amorphous phase (Nakagawa, 1973) in a given temperature range.

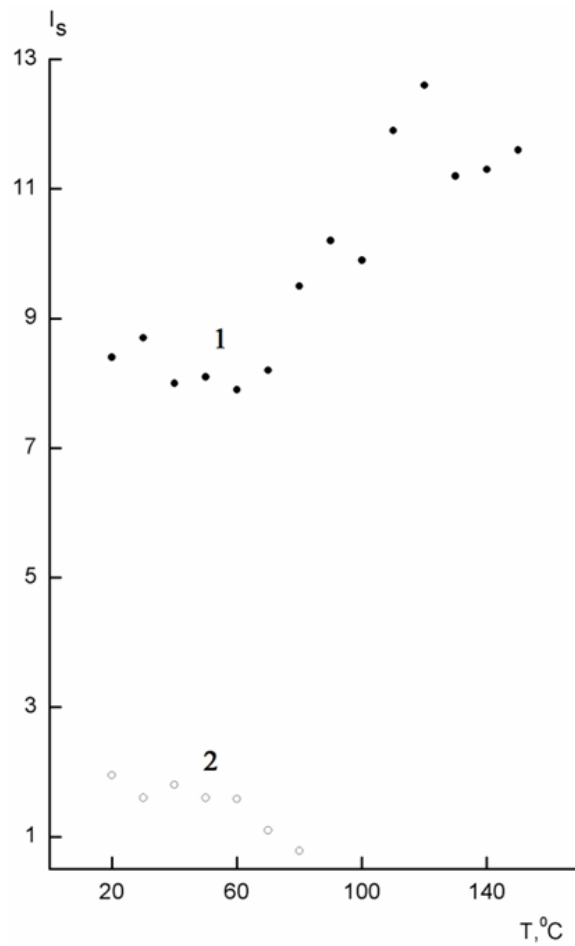

a)

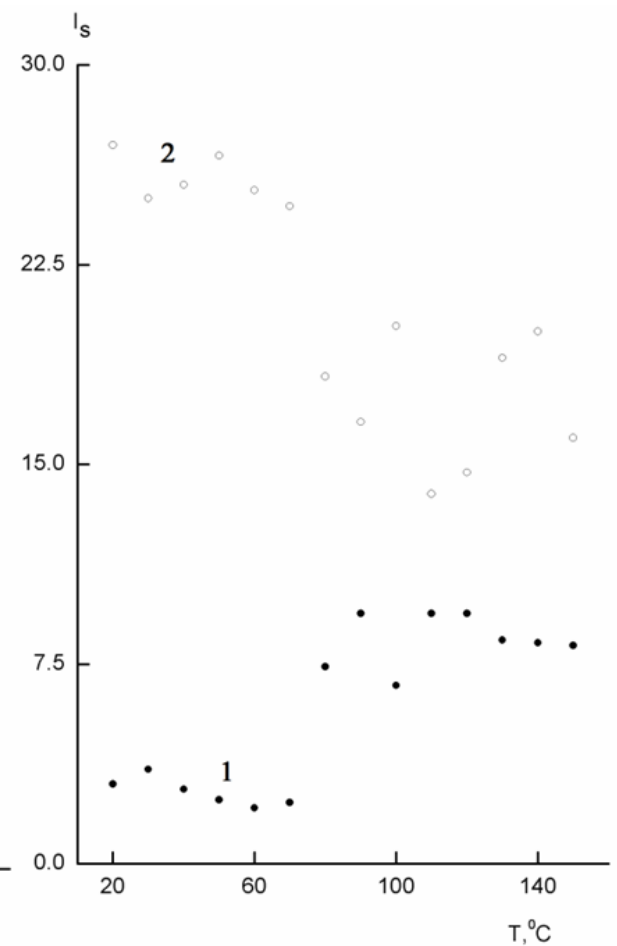

b)

Figure 12. Temperature dependences of the integral intensity of the symmetric (a) and asymmetric (b) stretching vibrations bands; low-frequency (1) and high-frequency (2) components

The effect of temperature on intermolecular interactions is shown in Figure 12. This data is pointed out the occurrence of unharmonious in methylene vibrational state. It is known, that the unharmonicity constant for the anti-symmetric X-H stretching vibrations are much stronger, than that for the symmetric modes (Hollas, 1987). While the symmetric mode fine structure disappears at $80^{\circ} \mathrm{C}$ (Figure 12a), for the anti-symmetric mode (Figure $12 b)$ it remains up to the melting point. Similar conclusion can be drawn on analyzing the spectroscopic data of overtones in the "premelting" in nylon 12 (Ozaki, 1997) or at phase transition in the low molecular n-alkanes (Zerbi, 1981). One of the reasons for this effect is obviously associated with a decrease in the regular conformation chains segments lengths (Kumpanenko, 1981). Actually, for PVDF and its copolymers, with the chains being predominantly planar zigzag conformation, the increase of the "defect" conformations ratio with temperature is observed (Wang, 1988; Nalva, 1995; Kochervinskii, 1996).

As noted above, since the polymers are strongly polar, the influence of electrostatic interactions on the spectral characteristics of absorption bands can be noted (Volkenstein, 1937). The bands of stretching vibrations of methylene groups in the polyethylene (PE) are characterized by a doublet $2850 ; 2920 \mathrm{~cm}^{-1} . \mathrm{CF}_{2}$ and $\mathrm{CH}_{2}$ interchange (idealized PVDF molecule) leads to a shift of the doublet in the $2980 ; 3020 \mathrm{~cm}^{-1}$ region (Wang, 1988; Nalva, 1995; Kochervinskii, 1999; Kobayashi, 1975). That is, strongly polar $\mathrm{CF}_{2}$ group's occurrence leads to a strong (100 and more $\left.\mathrm{cm}^{-1}\right)$ "blue" shift of the frequency. In this connection, methylene groups stretching vibrations characteristics changes with temperature rise is connected with local changes in polymer chains 
microstructure. These changes should lead to the local electrostatic field changes.

As noted above, in real polymers is always present a certain amount of ionic impurities, which can be called "quasifree" charges. If their concentration is high enough, the local electric field $E_{i}$ near the site of the chain should take into account the field produced by ionogenic impurities. Since we have investigated the ferroelectric copolymer, it is necessary to take into account the specifics of its structure. One of the ferroelectric properties is the presence of domains with the spontaneous polarization. The presence of the last, lead to the localize ionic impurities at the traps formed by the polar planes of domains. Validity of this hypothesis is testified by the following facts. In PVDF and its copolymers revealed the giant dielectric dispersion, which may be associated with the relaxation of space charge (Kochervinskii, Malyshkina, Markin, \& Gavrilova, 2007; Kochervinskii, Malyshkina, Gavrilova, Sulyanov, \& Bessonova, 2007; Kochervinskii \& Malyshkina, 2010). According to these works, "quasi-free" charges begin freeing in the temperature of the secondary transition. It is clear that such a process should change the local field of the light wave. Therefore, for the copolymer we investigated the significant changes in the absorption bands characteristics are in the secondary transition area.

\section{Conclusion}

Structural changes in the isotropic copolymer films of vinylidenefluoride with tetrafluoroethylene in a metastable state have been studied by the DSK, the X-ray diffraction and IR. Recrystallization processes during these films heating results in the temperature dependence change both of the dielectric constant and conductivity. Methylene groups symmetric and anti-symmetric vibrations components temperature changes analysis show, that these bands parameters are very sensitive both to the chains relaxation in the "rigid amorphous phase" and to the melting imperfect crystals formed during secondary crystallization. First proposed to use for the description of spectroscopic characteristics of the considered polymers (with high dipole moment) rules observed in strongly polar liquids. From these positions is shown to explain the unusual "blue" shift of valence oscillation $\mathrm{C}-\mathrm{H}$ groups in the region of the phase transition.

The works is supported by RFBR grant 10-03-00914.

\section{References}

Bachmann, M. A., Gordon, W. L., Koenig, J. L., \& Lando, W. L. (1979). An Infrared study of phase-III poly(vinylidenefluoride). J. Appl. Phys., 50(10), 6105-6112. http://dx.doi.org/10.1063/1.325780

Bahshiev, N. G. (1972). Spectroscopy of the Intermolecular Interactions (Russia). Leningrad: Science.

Bretzlaff, R. S., \& Wool, R. (1981). Temperature effect in quasiharmonic infrared bands of stressed polymers. $J$. Appl. Phys., 52(10), 5964-5969. http://dx.doi.org/10.1063/1.328527

Bretzlaff, R. S., \& Wool, R. (1983). Frequency shifting and asymmetry in infrared bands of stressed polymers. Macromolecules, 16, 1907-1917. http://dx.doi.org/10.1021/ma00246a019

Bronnikov, S. V., Vettegren, V. I., \& Frenkel, S. Y. (1996). Kinetics of deformation and relaxation in higly oriented polymers. Adv. Polym. Sci., 125, 103-146. http://dx.doi.org/10.1007/BFb0071156

Bulanin, M. O., \& Tonkov, M. V. (1964). Optic Spectrosc (Russia), 16(3), 429.

Bulanin, M. O., Orlova, N. D., \& Shchepkin, D. I. (1965). Optic Spectrosc. (Russia), 19(5), 731.

Hollas, J. M. (1987). Modern Spectroscopy. Chichester. UK: John Wiley \& Sons.

Horiuchi, T., Matsushige, K., \& Takemura, T. (1986). Intermediate structure at the ferro-to paraelectric phase transition of VDF/TrFE copolymer $(54 / 46 \mathrm{~mol} \%)$. Jpn. J. Appl. Phys., 25(6), L465-L467. http://dx.doi.org/10.1143/JJAP.25.L465

Ikeda, S., \& Wada, Y. A. (1984). Theoretical analysis of dielectric constant of copolymer of vinylidenefluoride and trifluoroethylene near its ferroelectric transition temperature. Ferroelectrics, 57, 85-88.

Kobayashi, М., Таshико, К., \& Tadokoro, Н. (1975). Molecular vibrations of three crystalline forms of poly(vinylidenefluoride). Macromolecules, 8(2), 158-171. http://dx.doi.org/10.1021/ma60044a013

Kochervinskii, V. V. (1994). Properties and applications of fluorine contain films with piezo- and pyroactivity. Russ. Chem. Rev., 63(4), 367.

Kochervinskii, V. V. (1996). Structure and properties of bulk polyvinyliden fluorine and systems based on it. Russ. Chem. Rev., 65(10), 865.

Kochervinskii, V. V. (1999). Ferroelectric properties of vinylidenefluoride based polymers. Russ. Chem. Rev., 68(10), 821. 
Kochervinskii, V. V. (2009). New electrostriction materials based on organic polymers: A review. Crystallography Reports. Suppl., 54(7), 1146-1171.

Kochervinskii, V. V., \& Murasheva, E. M. (1991). Microstructure and ferroelectric of vinyliden fluoride and tetrafluoretylen composition 71/29. Polymer Sci. A. (Russia), 33(10), 1967.

Kochervinskii, V. V., \& Suljanov, S. N. (2006). Structure formation in crystallizing ferroelectric polymers. Phys. Sol. State (Russia), 48(6), 1079.

Kochervinskii, V. V., Bessonova, N. P., Malyshkina, I. A., Sulyanov, S. N., \& Dembo, K. A. (2011). Effect of Recrystallization on the Molecular Mobility in Vinylidenefluoride-Hexafluoropropylene Copolymer. $J$. Appl. Polym. Sci., 120(1), 13-20. http://dx.doi.org/10.1002/app.32993

Kochervinskii, V. V., Kozlova, N. V., Khnykov, A. Yu., Shcherbina, M. A., Sulyanov, S. N., \& Dembo, K. A. (2010). The features of structure formation and electrophyscal properties of polyvinylidenefluoride crystalline ferroelectric polymers. J. Appl. Polym. Sci., 116, 695.

Kochervinskii, V. V., Malyshkina, I. A., Markin, G. V., \& Gavrilova, N. D. (2007). Dielectric relaxation in vinyliden fluoride - hexafluoropropylene copolymers. J. Appl. Polym. Sci., 105(3), 1101. http://dx.doi.org/10.1002/app.26145

Kochervinskii, V. V., Sokolov, V. G., \& Zubkov, V. M. (1991). Influence of the molecular structure on characteristics dielectric hysteresis of polyvinylidenefluoride and their copolymers. Polymer Sci. A. (Russia), 33(3), 450.

Kochervinskii, V. V., Volkov, V. V., \& Dembo, K. A. (2006). The role of intrachain dipole interactions in the formation of the supramolecular structure of crystallizing ferroelectric polymers. Phys. Sol. State (Russia), 48(6), 1083. http://dx.doi.org/10.1134/S1063783406060205

Kochervinskii, V., \& Malyshkina, I. (2010). Peculiarities of high-temperatures dielectric relaxation in (vinylidenfluoride)-(hexafluoropropylene) copolymers. $J . \quad$ Non-Cryst. $\quad$ Solids., $356,564$. http://dx.doi.org/10.1016/j.jnoncrysol.2009.09.034

Kochervinskii, V., Malyshkina, I., Gavrilova, N., Sulyanov, S., \& Bessonova, N. (2007). Peculiarities of dielectric relaxation in poly(vinylidenfluoride) with different thermal history. J. Non-Cryst. Solids., 353, 4443. http://dx.doi.org/10.1016/j.jnoncrysol.2007.03.034

Koizumi, N., Yaikawa, N., \& Habuka, H. (1984). Dielectric behavior and ferroelectric transition of copolymers of vinylidenefluoride and trifluoroethylene. Ferroelectrics, 57(1-4), 99-119. http://dx.doi.org/10.1080/00150198408012756

Kumpanenko, I. V., \& Chukanov, N. V. (1981). Regularity band in the infrared spectra of polymers with violations of the periodicity of the structure. Uspehi Chem. (Russia), l(9), 1627.

Lovinger, A. J., Davis, D. D., Cais, R. E., \& Kometani, J. M. (1988).Compositional variation of the structure and solid-state transformations of vinylidene/tetrafluoroethylene copolymers. Macromolecules, 21, 78-83. http://dx.doi.org/10.1021/ma00179a017

Miyata, Y. (1988). Relaxation and transition of copolymers of vinylidenefluoride and trifluoroethylene with high trifluoroethylene content. Polymer J., 20(9), 771-783.

Nakagawa, K., \& Ishida, Y. (1973). Dielectric relaxations and molecular motions in poly(vinylidene fluoride) with crystal form II. Journal of Polymer Science Part B: Polymer Physics, 11(8), 1503-1533. http://dx.doi.org/10.1002/pol.1973.180110804

Nalva, H. S. (Ed.). (1995). Ferroelectric Polymers - Chemistry, Physics and Applications. New York: Marcel Dekker Inc.

Natesan, B., Xu, H., Ince, B. S., \& Cebe, J. (2004). Molecular relaxation of isotactic polystyrene: Real-time dielectric spectroscopy and X-ray scattering studies. Journal of Polymer Science Part B: Polymer Physics, 42(5), 777-789. http://dx.doi.org/10.1002/polb.10757

Neidhoffer, M., Beaume, F., Ibos, L., Bernes, A., \& Lacabanne, C. (2004). Structural evolution of PVDF during storage or annealing. Polymer, 45(5), 1679-1688. http://dx.doi.org/10.1016/j.polymer.2003.12.066

Oka, Y., Murata, Y., \& Koizumi, N. (1986). Structute and Spontaneous Polarization in Fast-Quenched Copolymers of Vinylidenefluoride and Trifluoroethylene. Polymer Journal, 18(5), 417-427. http://dx.doi.org/10.1295/polymj.18.417 
Ozaki, Y., Liu, Y., \& Noda, I. (1997). Two-dimensional near-infrared correlation spectroscopy study of premelting behavior of nylon 12. Macromolecules, 30, 2391-2399. http://dx.doi.org/10.1021/ma961124s

Peng, Y., \& Wu, A. (2004). Two dimensional infrared correlation spectroscopic study on the structure changes of PVDF during the melting process. Polymer, 45, 5295-5299. http://dx.doi.org/10.1016/j.polymer.(2004).05.034

Rakov, A. V. (1962). Optic Spectrosc. (Russia), 13(3), 369.

Rinaldo, G., \& Botta, M. M. (1988). Effect of crystallization Temperature on the PhaseTransitions of P(VDF-TrFE) Copolymers. J. Polym. Sci. Polym. Phys., 36, 403-414.

Sechkarev, A. V. (1965). Optic Spectrosc. (Russia), 19(5), 721.

Shapiro, A., Mankowski, Z., \& Schmitt, N. (1982). Unusial Swelling Behavior of Films of Polyvinyl-and Polyvinylidene/Fluorides in Various Solvents. Journal of Polymer Science: Polymer Chemistry Edition, 20(7), 1791-1796. http://dx.doi.org/10.1002/pol.1982.170200712

Takahashi, Y., Kodama, H., Nakamura, M., Furukawa, T., \& Date, M. (1999). Antiferroelectric-like behavior of vinylidenefluoride/trifluoroethylene copolymers with low vinylidene fluoride content. Polymer Journal, 31(3), 263-267. http://dx.doi.org/10.1295/polymj.31.263

Tanaka. H., Yukawa, H., \& Nishi, T. (1988). Effect of Crystallization Condition on the Ferroelectric Phase Transition in Vinylidenefluoride/Trifluoroethylene Copolymers. Macromolecules, 21(8), 2469-2474.

Tashiko, K., Kobayashi, M., \& Tadokoro, H. (1981). Vibration spectra and disorder-order transition of poly(vinylidenefluoride) form III. Macromolecules, 14, 1757-1764. http://dx.doi.org/10.1021/ma50007a028

Tashiro, K., Takano, K., Kobayashi, M., Chatani, Y., \& Tadokoro, H. (1983). Phase transition at a temperature immediately below the melting point of polyvinylidenefluoride form I: Propagation for the ferroelectric Curie point. Polymer, 24(2), 199-204. http://dx.doi.org/10.1016/0032-3861(83)90133-7

Valiev, K. A. (1961). Optic Spectrosc. (Russia), 11(4), 465.

Valiev, K. A. (1963). Optic Spectrosc. SbN2 Molecular spectroscopy (Russia), Acad Nauk USSR, 98.

Vettegren, V. I., \& Kulik, V. B. (2009). Vusokomol. Soed A. (Russia), 51, 1411.

Vettegren, V. I., Titenkov, L. S., \& Bronnikov, S. V. (1992). Thermophysical properties of macromolecules in the block state. J. Therm. Analysis, 38, 1031-1045. http://dx.doi.org/10.1007/BF01979167

Volkenstein, M. V. (1937). Usp. Fiz. Nauk. (Russia), 18, 153

Wang, T. T., Herbert, J. M., \& Glass, A. M. (Eds.). (1988). The Application of Ferroelectric Polymers. Glasgow: Blackie.

Zbinden, R. (1964). Infrared Spectroscopy of High Polymers. New York - London: Academic press.

Zerbi, G., Magni, R., Gussoni, M., Moritz, K., Bigotto, \& Dirlikov, S. J. (1981). Molecular mechanics for phase transition and melting of $\mathrm{n}$ - alkanes: A spectroscopic study of molecular mobility of solid $\mathrm{n}$ - nonadecane The Journal of Chemical Physics, 75(7), 3175-3194. http://dx.doi.org/10.1063/1.442490

\section{Copyrights}

Copyright for this article is retained by the author(s), with first publication rights granted to the journal.

This is an open-access article distributed under the terms and conditions of the Creative Commons Attribution license (http://creativecommons.org/licenses/by/3.0/). 